\title{
DETERMINACY, TRANSVERSALITY AND LAGRANGE STABILITY
}

\author{
GO-O ISHIKA W A \\ Department of Mathematics \\ Hokkaido University \\ Sapporo 060-0810, Japan \\ E-mail: ishikawa@math.sci.hokudai.ac.jp
}

Dedicated to Professor Takuo Fukuda on his sixtieth birthday

1. Introduction. In the present article, as a continuation of [14], we give Arnol'dMather type characterisation of Lagrange stability for a class of singular Lagrange varieties, open Whitney umbrellas, via the transversality in isotropic jet spaces. Also the determinacy of isotropic map-germs by jets under Lagrange equivalence is considered. We give an example of Lagrange stable isotropic map-germ of corank one in itself and of corank two after the Lagrange projection. Lastly we mention open questions.

Let $X$ be an $n$-dimensional manifold, and $(M, \omega)$ a $2 n$-dimensional symplectic manifold with a symplectic form $\omega(n \geq 1)$. A $C^{\infty}$ mapping $f: X \rightarrow M$ is called isotropic if $f^{*} \omega=0$. Then $f$ is a Lagrange immersion off the singular locus $\Sigma(f)=\{x \in X \mid$ $f$ is not immersive at $x\}$.

The main object we are studying is a special class of singularities of isotropic mappings, namely, the open Whitney umbrellas, which are first recognised by Arnol'd, Givental $^{\prime}$ and Zakalyukin $[6,7]$.

We are mainly interested in the case $M=T^{*} Y$, the cotangent bundle over an $n$-manifold $Y$, endowed with the symplectic form $\omega=d \theta_{Y}$, the exterior differential of the Liouville 1-form $\theta_{Y}$ on $T^{*} Y$. Consider the canonical Lagrange projection $\pi: T^{*} Y \rightarrow Y$. Then singularities of Lagrange projections of Lagrange submanifolds are called Lagrange singularities. The study of Lagrange singularities is reduced by Hörmander and $\mathrm{Arnol}^{\prime} \mathrm{d}$ to the theory of deformations of functions by means of generating families of Morse type. Based on this reduction, Lagrange singularities are studied extensively. See [1, 26, 4].

An open Whitney umbrella is obtained as a component of a singular Lagrange variety

1991 Mathematics Subject Classification: Primary 58C27; Secondary 58F05.

The paper is in final form and no version of it will be published elsewhere. 
induced by a non-Morse generating family. For this direction, see [15, 27]. In this paper we study singularities of Lagrange projections of open Whitney umbrellas, from the view point of Thom-Mather's theory of differentiable mapping.

After Thom's work, Mather, in the series of papers [18], gives the theory on $C^{\infty}$-stable mappings. Restricting ourselves to local $C^{\infty}$ theory, we recall the following results due to Mather on $C^{\infty}$ map-germs $f:\left(\mathbf{R}^{n}, 0\right) \rightarrow\left(\mathbf{R}^{p}, 0\right)$ :

(A) Infinitesimal characterisation of stable map-germs.

(B) Determinacy: If $f$ is stable then $f$ is $(p+1)$-determined.

(C) Classification by $\mathbf{R}$-algebras of stable map-germs. Construction of a stable germ of given algebra type.

(D) Characterisation of stability by transversality.

(E) Determination of "nice range" where stable maps are generic.

Then we are naturally led to the question: Are there analogies to Thom-Mather's theory, for Lagrange stable projections of open Whitney umbrellas?

A germ of submersion $\pi^{\prime}:\left(M, y_{0}\right) \rightarrow\left(Y, z_{0}\right)$ is called a Lagrange projection if all fibres of $\pi^{\prime}$ are Lagrange submanifolds, in other word, if any pairs of components of $\pi^{\prime}$ are Poisson commutative.

Consider a pair $(f, \pi)$ (resp. $\left.\left(f^{\prime}, \pi^{\prime}\right)\right)$ of map-germs $f:\left(X, x_{0}\right) \rightarrow\left(M, y_{0}\right)$ (resp. $\left.f^{\prime}:\left(X^{\prime}, x_{0}^{\prime}\right) \rightarrow\left(M^{\prime}, y_{0}^{\prime}\right)\right)$ and a Lagrange projection $\pi:\left(M, y_{0}\right) \rightarrow\left(Y, z_{0}\right)$ (resp. $\pi^{\prime}:$ $\left.\left(M^{\prime}, y_{0}^{\prime}\right) \rightarrow\left(Y^{\prime}, z_{0}^{\prime}\right)\right)$. Then $(f, \pi)$ and $\left(f^{\prime}, \pi^{\prime}\right)$ are called Lagrange equivalent if there exist a diffeomorphism-germ $\sigma:\left(X, x_{0}\right) \rightarrow\left(X^{\prime}, x_{0}^{\prime}\right)$, a symplectomorphism-germ $\tau:\left(M, y_{0}\right) \rightarrow$ $\left(M^{\prime}, y_{0}^{\prime}\right)$ and a diffeomorphism-germ $\bar{\tau}:\left(Y, z_{0}\right) \rightarrow\left(Y^{\prime}, z_{0}^{\prime}\right)$ such that $\tau \circ f=f^{\prime} \circ \sigma$ and that $\bar{\tau} \circ \pi=\pi^{\prime} \circ \tau$.

In [14], we show the equivalence of the "homotopical" Lagrange stability and the infinitesimal Lagrange stability. An isotropic map-germ $f:\left(X, x_{0}\right) \rightarrow\left(T^{*} Y, y_{0}\right)$ is homotopically Lagrange stable with respect to the standard Lagrange projection $\pi: T^{*} Y \rightarrow Y$, if any 1-parameter isotropic deformations $f_{t}$ of $f$ are trivialised under Lagrange equivalence, namely, if the pair $\left(f_{t}, \pi\right)$ and $(f, \pi)$ are Lagrange equivalent by families $\left(\sigma_{t}, \tau_{t}, \bar{\tau}_{t}\right)$.

Infinitesimal Lagrange stability is defined naturally in [14]. See also Section 3.

In this paper we define the Lagrange stability as follows: Roughly speaking, an isotropic map-germ $f:\left(X, x_{0}\right) \rightarrow\left(T^{*} Y, y_{0}\right)$ is Lagrange stable if, by any sufficiently small isotropic perturbations, the Lagrange equivalence class of $f_{x_{0}}$ is not removed. To formulate accurately, denote by $C_{I}^{\infty}(X, M)$ the space of $C^{\infty}$-isotropic mappings from $X$ to $M$, endowed with the Whitney $C^{\infty}$ topology. Then an isotropic map-germ $f:\left(X, x_{0}\right) \rightarrow\left(T^{*} Y, y_{0}\right)$ is Lagrange stable if, for any isotropic representative $f: U \rightarrow T^{*} Y$ of $f$, there exists a neighbourhood $W$ in $C_{I}^{\infty}(X, M)$ such that, for any $f^{\prime} \in W$, the original pair of germs $(f, \pi)$ is Lagrange equivalent to $\left(f_{x_{0}^{\prime}}^{\prime}, \pi\right)$ for some $x_{0}^{\prime} \in U$ (cf. [4], page 325).

To characterise the Lagrange stability by means of transversality, we recall the isotropic jet spaces [13]. Denote by $J_{I}^{r}(X, M)$ the set of $r$-jets of isotropic map-germs $f$ : $\left(X, x_{0}\right) \rightarrow\left(M, y_{0}\right)$ of corank at most one:

$$
J_{I}^{r}(X, M)=\left\{j^{r} f\left(x_{0}\right) \mid f:\left(X, x_{0}\right) \rightarrow\left(M, y_{0}\right) \text { isotropic, } \text { corank }_{x_{0}} f \leq 1\right\} .
$$

Then $J_{I}^{r}(X, M)$ is a submanifold of the ordinary jet space $J^{r}(X, M)([13])$. Moreover, 
for $z=j^{r} f\left(x_{0}\right) \in J_{I}^{r}(X, M), r$-jets of map-germs which are Lagrange equivalent to $f:\left(X, x_{0}\right) \rightarrow\left(M, y_{0}\right)$ form a submanifold of $J_{I}^{r}(X, M)$.

If $f: X \rightarrow M$ is an isotropic mapping of corank at most one, then the image of the $r$-jet section $j^{r} f: X \rightarrow J^{r}(X, M)$ is contained in $J_{I}^{r}(X, M)$. Then we regard $j^{r} f$ as a mapping to $J_{I}^{r}(X, M)$.

For a manifold-germ $\left(X, x_{0}\right)$, we denote by $E_{X, x_{0}}$ the $\mathbf{R}$-algebra consisting of $C^{\infty}$ function-germs $\left(X, x_{0}\right) \rightarrow \mathbf{R}$, and by $m_{X, x_{0}}$ the unique maximal ideal of $E_{X, x_{0}}$. If the base point $x_{0}$ is clear in the context, we abbreviate $E_{X, x_{0}}$ and $m_{X, x_{0}}$ to $E_{X}$ and $m_{X}$ respectively.

Now set

$$
r_{0}=\inf \left\{r \in \mathbf{N} \mid f^{*} E_{T^{*} Y} \cap m_{X}^{r+2} \subset f^{*} m_{T^{*} Y}^{n+3}\right\} .
$$

Then, by Artin-Rees type theorem, $r_{0}$ is a finite positive integer, determined by $n$ and $k$, the type of the open Whitney umbrella. Actually $r_{0}$ depends only on the right-left equivalent class of $f$.

The purpose of this paper is to show the following result, which is an analogue to the points (A) and (D):

Theorem 1.1. Let $\operatorname{dim} X=\operatorname{dim} Y=n$ and $f:\left(X, x_{0}\right) \rightarrow\left(T^{*} Y, f\left(x_{0}\right)\right)$ an open Whitney umbrella. Then the following conditions are equivalent to each other for $r \geq r_{0}$ :

(s) $f$ is Lagrange stable.

(hs) $f$ is homotopically Lagrange stable.

(is) $f$ is infinitesimally Lagrange stable.

(a) $f^{*} E_{T^{*} Y}$ is generated by $1, p_{1} \circ f, \ldots, p_{n} \circ f$ as $E_{Y}$-module via $(\pi \circ f)^{*}$.

$\left(\mathrm{a}^{\prime}\right) f^{*} E_{T^{*} Y} /(\pi \circ f)^{*} m_{Y} f^{*} E_{T^{*} Y}$ is generated by $1, p_{1} \circ f, \ldots, p_{n} \circ f$ over $\mathbf{R}$.

$\left(\mathrm{a}_{r}^{\prime \prime}\right) f^{*} E_{T^{*} Y} /\left\{(\pi \circ f)^{*} m_{Y} f^{*} E_{T^{*} Y}+f^{*} E_{T^{*} Y} \cap m_{X}^{r+2}\right\}$ is generated by $1, p_{1} \circ f, \ldots, p_{n} \circ f$ over $\mathbf{R}$.

$\left(\mathrm{t}_{r}\right)$ The jet extension $j^{r} f:\left(X, x_{0}\right) \rightarrow J_{I}^{r}\left(X, T^{*} Y\right)$ is transversal to the Lagrange equivalence class of $j^{r} f\left(x_{0}\right)$.

For the notation, see [14] and Sections 2, 3.

In the case $f$ is a Lagrange immersion, the condition $\left(\mathrm{a}^{\prime}\right)$ is equivalent to the one that a generating family of $f$ is $R_{+}$-versal [4]. In this case we see $r_{0}=n+1$.

Corollary 1.2 (Arnol'd, Tsukada). A Lagrange immersion-germ is Lagrange stable if and only if its generating family is $R_{+}$-versal.

This is clearly formulated in [4], while the explicit proof is omitted, as far as the author knows, T. Tsukada has given an explicit proof in his unpublished work [23]. In the proof by Tsukada, the perturbations of Lagrange immersions and those of their generating families are studied explicitly, to show the equivalence of Lagrange stability and stability of the generating families as unfoldings of functions. In our proof, Lagrange stability is directly described, in the natural way, by the transversality in the space of isotropic jets. 
To establish the description, we need the determinacy result for isotropic map-germs. On the ordinary theory of determinacy of map-germs, refer to the excellent survey [24]. Here we treat its isotropic counterpart.

An isotropic map-germ $f:\left(X, x_{0}\right) \rightarrow\left(T^{*} Y, y_{0}\right)$ is called Lagrange $r$-determined if, for any Lagrange projection $\pi^{\prime}:\left(T^{*} Y, y_{0}\right) \rightarrow Y$ with $j^{r} \pi^{\prime}\left(y_{0}\right)=j^{r} \pi\left(y_{0}\right)$, two pairs $\left(f, \pi^{\prime}\right)$ and $(f, \pi)$ are Lagrange equivalent. An isotropic map-germ $f:\left(X, x_{0}\right) \rightarrow\left(T^{*} Y, y_{0}\right)$ is called strictly Lagrange $r$-determined if, for any isotropic map-germ $f:\left(X, x_{0}\right) \rightarrow\left(T^{*} Y, y_{0}\right)$ with $j^{r} f^{\prime}\left(x_{0}\right)=j^{r} f\left(x_{0}\right),\left(f^{\prime}, \pi\right)$ and $(f, \pi)$ are Lagrange equivalent.

We easily see that, if $f$ is strictly Lagrange $r$-determined, then $f$ is Lagrange $r$-determined. In the case $f$ is a Lagrange immersion, these two notions coincide.

For the point (B), we show the following result, which seems to be a special case of a theorem due to Givental' ([7], Corollary 1, "sufficient jet theorem"):

TheOrem 1.3. Let $\operatorname{dim} X=\operatorname{dim} Y=n$. If an open Whitney umbrella $f:\left(X, x_{0}\right) \rightarrow$ $\left(T^{*} Y, y_{0}\right)$ is infinitesimally Lagrange stable, then $f$ is Lagrange $(n+1)$-determined and $f$ is strictly Lagrange $r_{0}$-determined.

Since no explicit proof is given in [7], we give a proof to assure ourselves.

For analogies to the points (C) and (E), see Section 6.

In the next section we recall the objects, open Whitney umbrellas. Theorem 1.3 is proved in Section 3, with recalling the notion of infinitesimal Lagrange stability. In Section 4 , we describe the transversality in the isotropic jet space, as the infinitesimal Lagrange stability up to finite order. Theorem 1.1 is proved in Section 5 .

For an isotropic map-germ $f:\left(X, x_{0}\right) \rightarrow\left(T^{*} Y, y_{0}\right)$, the corank of $\pi \circ f:\left(X, x_{0}\right) \rightarrow$ $\left(Y, \pi\left(y_{0}\right)\right)$ at $x_{0}$ is called $L$-corank of $f$. The classification of Lagrange stable open Whitney umbrellas with $L$-corank $\leq 1$ is given explicitly [27, 12]. In Section 6, we give an example $f:\left(\mathbf{R}^{5}, 0\right) \rightarrow\left(T^{*} \mathbf{R}^{5}, 0\right)$ of Lagrange stable open Whitney umbrella with $L$-corank 2 . Such example seems to have never been given in any literature so far.

The author would like to thank I. A. Bogaevski, S. Izumiya, S. Janeczko and V. M. Zakalyukin for valuable comments and helpful encouragement.

2. Open Whitney umbrellas. We recall the definition given in [14].

The local model of an open Whitney umbrella $f=f_{n, k}:\left(\mathbf{R}^{n}, 0\right) \rightarrow\left(T^{*} \mathbf{R}^{n}, 0\right)$ of type $k\left(0 \leq k \leq\left[\frac{n}{2}\right]\right)$ is concretely given by $q_{1} \circ f=x_{1}, \ldots, q_{n-1} \circ f=x_{n-1}$,

$$
\begin{gathered}
q_{n} \circ f=\frac{x_{n}^{k+1}}{(k+1) !}+x_{1} \frac{x_{n}^{k-1}}{(k-1) !}+\ldots+x_{k-1} x_{n}(=: u), \\
p_{n} \circ f=x_{k} \frac{x_{n}^{k}}{k !}+\ldots+x_{2 k-1} x_{n}(=: v),
\end{gathered}
$$

and

where $\frac{\partial(v, u)}{\partial\left(x_{i}, x_{n}\right)}$ is the Jacobian.

$$
p_{i}=\int_{0}^{x_{n}} \frac{\partial(v, u)}{\partial\left(x_{i}, x_{n}\right)} d x_{n}, \quad 1 \leq i \leq n-1,
$$


Remark that $f_{n, k}$ is isotropic, that is, $f_{n, k}^{*} \omega=0$, where $\omega=\sum_{i=1}^{n} d p_{i} \wedge d q_{i}$ is the standard symplectic form on $T^{*} \mathbf{R}^{n}$. Moreover, $f_{n, k}$ is a Lagrange immersion if and only if $k=0$. If $k \neq 0$, then the singular locus of $f_{n, k}$ is given by $\left\{\frac{\partial u}{\partial x_{n}}=\frac{\partial v}{\partial x_{n}}=0\right\}$ and, therefore, has codimension two.

In general a $C^{\infty}$ map-germ $f:\left(X, x_{0}\right) \rightarrow\left(M, y_{0}\right)$ is called an open Whitney umbrella of type $k$ if $f$ is symplectically equivalent to $f_{n, k}$, namely, if there exist a diffeomorphismgerm $\sigma:\left(X, x_{0}\right) \rightarrow\left(\mathbf{R}^{n}, 0\right)$ and a symplectomorphism-germ $\tau:\left(M, y_{0}\right) \rightarrow\left(T^{*} \mathbf{R}^{n}, 0\right)$ such that $\tau \circ f=f_{n, k} \circ \sigma$.

Thus Lagrange immersions are naturally generalised to open Whitney umbrellas: In [14], we introduce the notion of symplectic stability and characterise open Whitney umbrellas as symplectically stable isotropic map-germs of corank at most one.

In [14], Proposition 4.1, it is proved that if $f:\left(X, x_{0}\right) \rightarrow\left(T^{*} Y, y_{0}\right)$ is an open Whitney umbrella, then the ramification module

$$
R_{f}=\left\{e \in E_{X} \mid d e \in\left\langle d\left(p_{1} \circ f\right), \ldots, d\left(p_{n} \circ f\right), d\left(q_{1} \circ f\right), \ldots, d\left(q_{n} \circ f\right)\right\rangle_{E_{X}}\right\},
$$

is equal to the image $f^{*} E_{T^{*} Y}$ of the pull-back $f^{*}: E_{T^{*} Y} \rightarrow E_{X}$. Moreover, we see the following (cf. [11]), which is needed later:

Lemma 2.1. Let $f=f_{n, k}:\left(\mathbf{R}^{n}, 0\right) \rightarrow\left(T^{*} \mathbf{R}^{n}, 0\right)$ be the local model of the open Whitney umbrella of type $k$, and denote by $m\left(R_{f}\right)$ the unique maximal ideal of $R_{f}$. Then $x_{1}, \ldots, x_{n-1}, u, v, p_{i} \circ f(1 \leq i \leq 2 k-1)$ form a basis of the "Zariski cotangent space" $m\left(R_{f}\right) / m\left(R_{f}\right)^{2} \cong f^{*} m_{T^{*} Y} / f^{*} m_{T^{*} Y}^{2}$ over $\mathbf{R}$.

We call an isotropic map-germ $f:\left(X, x_{0}\right) \rightarrow\left(T^{*} Y, y_{0}\right)$ symplectically $r$-determined if any isotropic map-germ $f^{\prime}$ with $j^{r} f^{\prime}\left(x_{0}\right)=j^{r} f\left(x_{0}\right)$ is symplectically equivalent to $f$.

Clearly a Lagrange immersion is symplectically 1-determined. Similarly we have

Lemma 2.2. Let $f:\left(X, x_{0}\right) \rightarrow\left(M, y_{0}\right)$ be an open Whitney umbrella of type $k$, $0 \leq k \leq\left[\frac{n}{2}\right]$. Then $f$ is symplectically $(k+1)$-determined. In particular, an open Whitney umbrella is symplectically $n$-determined.

Proof. By [10], the condition that $f$ is an open Whitney umbrella of type $k$ is described by the transversality of $k$-jet extension of (some components of) $f$. Therefore the condition depends only on its $(k+1)$-jet at the base point. This implies the result.

3. Determinacy. The following is a fundamental fact we need (cf. [7]):

LEMMA 3.1. Let $r \geq 0$, and $\pi^{\prime}:\left(T^{*} \mathbf{R}^{n}, 0\right) \rightarrow\left(\mathbf{R}^{n}, 0\right)$ be a Lagrange projection with $j^{r} \pi^{\prime}(0)=j^{r} \pi(0)$, for the standard projection $\pi: T^{*} \mathbf{R}^{n} \rightarrow \mathbf{R}^{n}$. Then there exists a symplectic diffeomorphism $\tau:\left(T^{*} \mathbf{R}^{n}, 0\right) \rightarrow\left(T^{*} \mathbf{R}^{n}, 0\right)$ such that $\pi^{\prime}=\pi \circ \tau$ and $j^{r} \tau(0)=$ $j^{r} \operatorname{id}(0)$.

Proof. The result for the case $r=0$ is just the Darboux theorem for Lagrange projections ([4], Theorem 18.4). The proof for arbitrary $r$ follows from that for $r=0$.

Therefore we see 
LEMMA 3.2. Let $r \geq 1$ and $f:\left(X, x_{0}\right) \rightarrow\left(T^{*} Y, y_{0}\right)$ be an isotropic map-germ. Then the following statements are equivalent to each other:

(1) For any symplectic diffeomorphism $\tau:\left(T^{*} Y, y_{0}\right) \rightarrow\left(T^{*} Y, y_{0}\right)$ with $j^{r} \tau\left(y_{0}\right)=$ $j^{r} \mathrm{id}\left(y_{0}\right), \tau \circ f$ is Lagrange equivalent to $f$ with respect to $\pi$.

(2) For any Lagrange projection $\pi^{\prime}:\left(T^{*} Y, y_{0}\right) \rightarrow Y$ with $j^{r} \pi^{\prime}\left(y_{0}\right)=j^{r} \pi\left(y_{0}\right),\left(f, \pi^{\prime}\right)$ is Lagrange equivalent to $(f, \pi)$.

We recall that the infinitesimal Lagrange stability of $f$ is written as

$$
V I_{f}=t f\left(V_{X}\right)+w f\left(V L_{T^{*} Y}\right) .
$$

We denote by $V I_{f}$ the set of infinitesimal isotropic deformations of $f$. Remark that the symplectic structure on $T^{*} Y$ induces the isomorphism, therefore a diffeomorphism $T\left(T^{*} Y\right) \cong T^{*}\left(T^{*} Y\right)$. Besides, $T^{*}\left(T^{*} Y\right)$ has the natural symplectic structure $\omega=d \theta_{T^{*} Y}$, where $\theta_{T^{*} Y}$ is the Liouville 1-form on $T^{*}\left(T^{*} Y\right)$. Therefore we have naturally a symplectic structure $\tilde{\omega}=d \tilde{\theta}_{T^{*} Y}$ on $T\left(T^{*} Y\right)$, via the above isomorphism. An infinitesimal deformation $v:\left(X, x_{0}\right) \rightarrow T\left(T^{*} Y\right)$ of $f$ is called isotropic, if the pull-back 2-form $v^{*} \tilde{\omega}=0$. $V_{X}$ means the set of germs of vector fields $\xi:\left(X, x_{0}\right) \rightarrow T X$ along the identity. Moreover, we denote by $V L_{T^{*} Y}$ the set of infinitesimal Lagrange diffeomorphisms, namely, the set of germs of Hamiltonian vector fields $\eta:\left(T^{*} Y, y_{0}\right) \rightarrow T\left(T^{*} Y\right)$ with affine Hamiltonian of type $a_{0}(q)+a_{1}(q) p_{1}+\ldots+a_{n}(q) p_{n}$. Then we set $t f(\xi)=f_{*} \xi$ and $w f(\eta)=\eta \circ f$, for $\xi \in V_{X}, \eta \in V L_{T^{*} Y}$.

If $v \in V I_{f}$, then $d\left(v^{*} \tilde{\theta}_{T^{*} Y}\right)=v^{*} \tilde{\omega}=0$. Then there exists a function-germ $e \in E_{X}=$ $\left\{\left(X, x_{0}\right) \rightarrow \mathbf{R}\right\}$ such that $d e=v^{*} \tilde{\theta}_{T^{*} Y}$. We call $e$ a generating function of $v$. Then

$$
R_{f}=\left\{e \in E_{X} \mid e \text { is a generating function for some } v \in V L_{f}\right\}
$$

is a sub-R-algebra of $E_{X}$ containing $f^{*} E_{T^{*} Y}$.

Notice that $V I_{f}$ has an $E_{T^{*} Y}$-module structure and $V L_{T^{*} Y}$ has an $E_{Y}$-module structure [14]. In particular, for $h \in E_{T^{*} Y}$ and $v \in V I_{f}$, the $E_{T^{*} Y}$-multiplication is defined by

$$
h * v=h \circ f \cdot v-e \cdot X_{h} \circ f,
$$

where $\cdot$ is the pointwise multiplication, $e$ is the generating function of $v$ with $e\left(x_{0}\right)=0$, and $X_{h}$ is the germ of Hamiltonian vector field with Hamiltonian $h$ so that $i_{X_{h}} \omega=-d h$.

Set $M=T^{*} Y$. Since $f$ is an open Whitney umbrella, we see $R_{f}=f^{*} E_{M}$. Remark that $R_{f}$ is an $E_{M}$-module via $f^{*}$ and an $E_{Y}$-module via $(\pi \circ f)^{*}$. Then $m_{M} R_{f}=m\left(R_{f}\right)=$ $f^{*}\left(m_{M}\right)$.

LEMma 3.3. Let $f:\left(X, x_{0}\right) \rightarrow\left(T^{*} Y, y_{0}\right)$ be an infinitesimally Lagrange stable open Whitney umbrella. Then we have

(1) $m_{M}^{n+1} R_{f} \subset m_{Y} R_{f}$

(2) If $\pi^{\prime}:\left(T^{*} Y, y_{0}\right) \rightarrow Y$ is a Lagrange projection with $j^{n} \pi^{\prime}\left(y_{0}\right)=j^{n} \pi\left(y_{0}\right)$, then $f$ is infinitesimally Lagrange stable also with respect to $\pi^{\prime}$.

(3) $m_{T^{*} Y}^{n+2} V I_{f} \subset \operatorname{tf}\left(m_{X} V_{X}\right)+w f\left(m_{Y} V L_{T^{*} Y}\right)$.

Proof. (1) Suppose $f$ is infinitesimally Lagrange stable, that is, $V I_{f}=\operatorname{tf}\left(V_{X}\right)+$ $w f\left(V L_{T^{*} Y}\right)$. Set $Q_{f}=f^{*} E_{T^{*} Y} / m_{Y} f^{*} E_{T^{*} Y}=R_{f} / m_{Y} R_{f}$. Then $Q_{f}$ is generated by 
$1, p_{1} \circ f, \ldots, p_{n} \circ f$ over $\mathbf{R}$ by the equivalence of (is) and (a) ([14], Theorem 1.2). Therefore $\operatorname{dim}_{\mathbf{R}} Q_{f} \leq n+1$. Then considering the sequence of $E_{M}$-modules,

$$
Q_{f} \supset m_{M} Q_{f} \supset m_{M}^{2} Q_{f} \supset \ldots \supset m_{M}^{n+1} Q_{f},
$$

we see that $m_{M}^{n+1} Q_{f}=0$ and that $m_{M}^{n+1} R_{f} \subset m_{Y} R_{f}$, using Nakayama's lemma.

(2) Take a symplectic diffeomorphism $\tau$ as in Lemma 3.1 such that $\pi^{\prime}=\pi \circ \tau$. Set $g=\tau \circ f$. Then $R_{g}=R_{f}, q_{i} \circ g-q_{i} \circ f \in m_{M}^{n+1} R_{f}$ and $p_{j} \circ g-p_{j} \circ f \in m_{M}^{n+1} R_{f}$. Then by (1), $m_{Y} R_{g}=m_{Y} R_{f}$, with respect to $\pi$. Thus the condition (a) is satisfied also for $g$. Thus $g$ is Lagrange stable with respect to $\pi$, and therefore $f$ is Lagrange stable with respect to $\pi^{\prime}$.

(3) Take $v \in m_{M}^{n+2} V I_{f}$. Then $v$ has a generating function $e \in m_{M}^{n+3} R_{f}$. By (1), we see $m_{M}^{n+3} R_{f} \subset m_{Y} m\left(R_{f}\right)^{2}$. Therefore we have

$$
e=\left(\sum_{i=1}^{s} a_{i}(q) b_{i}(p, q)\right) \circ f
$$

for some $a_{i} \in m_{Y}$ and affine functions $b_{i} \in m_{T^{*} Y}$ with respect to $\pi$-fibres satisfying $b_{i} \circ f \in m\left(R_{f}\right)^{2}, 1 \leq i \leq n$. Set $h=\sum_{i=1}^{s} a_{i} b_{i}$ and consider the Hamiltonian vector field $X_{h}$ with the Hamiltonian $h$ on $T^{*} Y$. Then $X_{h} \in m_{Y} V L_{T^{*} Y}$, and $v-w f\left(X_{h}\right)$ has the generating function 0 . Therefore, by [14] Lemma 4.3, there exists $\xi \in V_{X}$ such that $v-w f\left(X_{h}\right)=t f(\xi)$. We show that $\xi(0)=0$.

If $f$ is an immersion, then the equality is clear. Assume $f$ is an open Whitney umbrella of type $k \geq 1$ and assume $\xi(0) \neq 0$. Then, with respect to the symplectic coordinates of normal forms for open Whitney umbrellas (Section 2), the coefficient of $\frac{\partial}{\partial q_{n}} \circ f$ of both sides of $v-w f\left(X_{h}\right)=t f(\xi)$ should be of order one. On the other hand, we see that, by Lemma 2.1, $\frac{\partial b_{i}}{\partial p_{n}}(0) \neq 0$ with respect to the coordinates of normal forms. Therefore the coefficient of $\frac{\partial}{\partial q_{n}} \circ f$ should be of order $\geq 2$. This leads to a contradiction, and we see that $\xi(0)=0$. Thus $v=t f(\xi)+w f\left(X_{h}\right)$ with $\xi \in m_{X} V_{X}, X_{h} \in m_{Y} V L_{T^{*} Y}$, and this proves (3).

Let $s$ be a positive integer. Consider the space $\operatorname{Sp}_{s}(n)$ of germs of symplectic diffeomorphisms $\left(T^{*} \mathbf{R}^{n}, 0\right) \rightarrow\left(T^{*} \mathbf{R}^{n}, 0\right)$ with identity $s$-jets. We need the following result on "connectivity" of $\operatorname{Sp}_{s}(n)$.

Proposition 3.4. Let $s \geq 1$. Then, for any pair $\tau_{0}, \tau_{1} \in \operatorname{Sp}_{s}(n)$, there exists a smooth family $\tau_{t}, 0 \leq t \leq 1$, connecting $\tau_{0}$ and $\tau_{1}$.

Pro of. Consider the graphs $\Gamma_{0}, \Gamma_{1}$ of $\tau_{0}, \tau_{1}$ respectively in $T^{*} \mathbf{R}^{n} \times T^{*} \mathbf{R}^{n}$, which are Lagrange submanifolds with respect to the symplectic form $\pi_{1}^{*} \omega-\pi_{2}^{*} \omega$. Take a Lagrange projection $\Pi: T^{*} \mathbf{R}^{n} \times T^{*} \mathbf{R}^{n} \rightarrow \mathbf{R}^{n} \times \mathbf{R}^{n}$ such that $\Gamma_{0}$, and therefore $\Gamma_{1}$, is mapped diffeomorphically. Then we can take generating functions $e_{0}, e_{1}$ of $\Gamma_{0}, \Gamma_{1}$ with respect to $\Pi$ such that $j^{s+1} e_{0}(0,0)=j^{s+1} e_{1}(0,0)$. Set $e_{t}=(1-t) e_{0}+t e_{1}$. Then the family of Lagrange submanifolds $\Gamma_{t}$ generated by $e_{t}$ corresponds to a family $\tau_{t} \in \operatorname{Sp}_{s}(n)$ connecting $\tau_{0}$ and $\tau_{1}$. 
We denote by $I_{f}^{s}$ the set of isotropic map-germs $f^{\prime}:\left(X, x_{0}\right) \rightarrow\left(M, y_{0}\right)$ with $j^{s} f^{\prime}\left(x_{0}\right)=$ $j^{s} f\left(x_{0}\right)$. To show the strict Lagrange determinacy we need the following:

Proposition 3.5. Let $s \geq k+1$. Let $f:\left(X, x_{0}\right) \rightarrow\left(M, y_{0}\right)$ be an open Whitney umbrella of type $k, 0 \leq k \leq\left[\frac{n}{2}\right], \operatorname{dim} X=n=\frac{1}{2} \operatorname{dim} M$. Then, for any pair $f_{0}, f_{1} \in I_{f}^{s}$, there exists a 1-parameter smooth family $f_{t} \in I_{f}^{s}, 0 \leq t \leq 1$, connecting $f_{0}$ and $f_{1}$.

Proof. We can assume that $f=f_{n, k}:\left(\mathbf{R}^{n}, 0\right) \rightarrow\left(T^{*} \mathbf{R}^{n}, 0\right)$. Then there exist a family of diffeomorphisms $\sigma_{t}:\left(\mathbf{R}^{n}, 0\right) \rightarrow\left(\mathbf{R}^{n}, 0\right)$ and $\bar{\tau}_{t}:\left(\mathbf{R}^{n}, 0\right) \rightarrow\left(\mathbf{R}^{n}, 0\right)$ such that $j^{s} \sigma_{t}(0)=j^{s} \operatorname{id}(0), j^{s} \bar{\tau}_{t}(0)=j^{s} \operatorname{id}(0)$ and that $\bar{\tau}_{1} \circ \pi \circ f_{0} \circ \sigma_{1}^{-1}=\pi \circ f_{1}$. We then set $\tau_{t}=\bar{\tau}_{t}^{-1 *}:\left(T^{*} \mathbf{R}^{n}, 0\right) \rightarrow\left(T^{*} \mathbf{R}^{n}, 0\right)$. Then $\tau_{t}$ is a family of symplectomorphisms with $j^{s} \tau_{t}(0)=j^{s} \operatorname{id}(0)$ and $\pi \circ \tau_{t}=\bar{\tau}_{t}$ (cf. Lemma 3.1). Consider the family $f_{t}^{\prime}=\tau_{t} \circ f \circ \sigma_{t}^{-1} \in I_{f}^{s}$. Then $\pi \circ f_{1}^{\prime}=\pi \circ f_{1}$. Take the generating functions $e^{\prime}$ and $e$ of $f_{1}^{\prime}$ and $f_{1}$ respectively so that $f_{1}^{\prime *} \theta=d e^{\prime}$ and $f_{1}^{*} \theta=d e$ with $e^{\prime}(0)=e(0)=0$. Then $j^{s+1} e^{\prime}(0)=j^{s+1} e(0)$. Now set $e_{t}=(1-t) e^{\prime}+t e$. Then there exists a family $f_{t}^{\prime \prime} \in I_{f}^{s}$ such that $f_{t}^{\prime \prime *} \theta=e_{t}$ and $\pi \circ f_{t}^{\prime \prime}=\pi \circ f_{1}^{\prime}\left(=\pi \circ f_{1}\right)$. Then $f_{0}^{\prime \prime}=f_{1}^{\prime}$ and $f_{1}^{\prime \prime}=f_{1}$. This proves the proposition.

\section{Proof of Theorem 1.3. Assume that}

$$
f:\left(X, x_{0}\right)=\left(\mathbf{R}^{n}, 0\right) \rightarrow\left(T^{*} Y, y_{0}\right)=\left(T^{*} \mathbf{R}^{n}, 0\right)
$$

is infinitesimally Lagrange stable and take $\tau \in \operatorname{Sp}_{n+1}(n)$. Then it suffices to show that, for the standard Lagrange projection $\pi:\left(T^{*} \mathbf{R}^{n}, 0\right) \rightarrow\left(\mathbf{R}^{n}, 0\right),(\tau \circ f, \pi)$ and $(f, \pi)$ are Lagrange equivalent. Set $g=\tau \circ f$. Then, by Proposition 3.4, there exists a smooth family $\tau_{t} \in \operatorname{Sp}_{n+1}(n)$ such that $\tau_{0}=$ id and $\tau_{1}=\tau$. Consider the family $f_{t}=\tau_{t} \circ f$ of isotropic map-germs. By Lemma $3.3(2)$, we see that each $f_{t}$ is infinitesimally Lagrange stable. Then, by Lemma 3.3 (3),

$$
m_{T^{*} Y}^{n+2} V I_{f_{t}} \subset t f_{t}\left(m_{X} V_{X}\right)+w f_{t}\left(m_{Y} V L_{T^{*} Y}\right) .
$$

Moreover this equality holds for vector fields smoothly depending on $t$ (see [14], Lemma 5.4). Therefore, for each $t_{0} \in[0,1]$, the family $f_{t}$ is trivialised under Lagrange equivalence $\left(\sigma_{t}, \tau_{t}^{\prime}\right)$ fixing base points; namely we have $f_{t_{0}}=\tau_{t}^{\prime} \circ f_{t} \circ \sigma_{t}, \tau_{t}$ is a Lagrange diffeomorphism, $\tau_{t}^{\prime}(0)=0$, and $\sigma_{t}(0)=0$. Thus $f=f_{0}$ and $g=\tau \circ f=f_{1}$ are Lagrange equivalent with respect to $\pi$.

Now we recall that $r_{0}$ is determined as the least positive integer satisfying $R_{f} \cap$ $m_{X}^{r_{0}+2} \subset f^{*} m_{T^{*} Y}^{n+3}$. For any $f^{\prime} \in I_{f}^{r_{0}}$, by Proposition 3.5, we connect $f^{\prime}$ and $f$ by a smooth family $f_{t} \in I_{f}^{r_{0}}$. Remark that $r_{0} \geq n+1$. Then, for the family $f_{t}$ of isotropic map-germs with $j^{r_{0}} f_{t}(0)=j^{r_{0}} f(0)$, we see, similarly as above,

$$
V I_{f_{t}} \cap m_{X}^{r_{0}+1} V_{f} \subset t f_{t}\left(m_{X} V_{X}\right)+w f_{t}\left(m_{Y} V L_{T^{*} Y}\right) .
$$

Thus $f_{t}$ is trivialised under Lagrange equivalence fixing base points.

4. Isotropic jets. Let $f:\left(X, x_{0}\right) \rightarrow\left(M, y_{0}\right)$ be an isotropic map-germ of corank at most one. We set

$$
V I_{f}^{s}=\left\{v \in V I_{f} \mid j^{s} v\left(x_{0}\right)=0\right\}=V I_{f} \cap m_{X}^{s+1} V_{f} \quad(s=0,1,2, \ldots) .
$$


Let $z \in J_{I}^{r}(n, 2 n)$. Define $\pi_{r}: V I_{f}^{0} \rightarrow T_{z} J^{r}(n, 2 n)$ as follows: For each $v \in V I_{f}^{0}$, take an isotropic deformation $f_{t}$ of $f$ with $v=\left.\frac{d f_{t}}{d t}\right|_{t=0}$, and set $\pi_{r}(v)=\left.\frac{d\left(j^{r} f_{t}(0)\right)}{d t}\right|_{t=0}$. Then the image of the linear map $\pi_{r}$ coincides with $\bar{T}_{z} J_{I}^{r}(n, 2 n)$.

Let $z \in J_{I}^{r}(n, 2 n)$ and $z=j^{r} f(0)$ for a $f:\left(\mathbf{R}^{n}, 0\right) \rightarrow\left(T^{*} \mathbf{R}^{n}, 0\right)$. Hereafter we set $X=\left(\mathbf{R}^{n}, 0\right), Y=\left(\mathbf{R}^{n}, 0\right)$ and $M=\left(T^{*} Y, 0\right)$. Then under the identification $T_{z} J^{r}(n, 2 n) \cong m_{X} V_{f} / m_{X}^{r+1} V_{f}$ we have

$$
T_{z} J_{I}^{r}(n, 2 n) \cong V I_{f}^{0} / V I_{f}^{r} .
$$

If we denote by $\mathcal{S}^{r} z\left(\right.$ resp, $\left.\mathcal{L}^{r} z\right)$ the orbit of $z$ under the symplectic equivalence (resp. Lagrange equivalence), we have

$$
\begin{gathered}
T_{z} \mathcal{S}^{r} z \cong V I_{f}^{0} /\left\{\left(t f\left(m_{X} V_{X}\right)+w f\left(m_{M} V H_{M}\right)\right) \cap V_{f}^{r}\right\}, \\
T_{z} \mathcal{L}^{r} z \cong V I_{f}^{0} /\left\{\left(t f\left(m_{X} V_{X}\right)+w f\left(m_{Y} V L_{M}\right)\right) \cap V_{f}^{r}\right\} .
\end{gathered}
$$

Set $z=j^{r} f\left(x_{0}\right)$. For $(w, v) \in T_{x_{0}} X \oplus V I_{f}$, take a curve $x_{t}$ in $X$ with the velocity vector $w$ at $t=0$ and take an isotropic deformation $f_{t}$ of $f$ with $v=\left.\frac{d f_{t}}{d t}\right|_{t=0}$ (cf. [14], Lemma 3.4), and define a linear map

$$
\Pi_{r}: T_{x_{0}} X \oplus V I_{f} \rightarrow T_{z} J^{r}(X, M),
$$

by

$$
\Pi_{r}(w, v)=\left.\frac{j^{r} d f_{t}\left(x_{t}\right)}{d t}\right|_{t=0} .
$$

Then $\Pi_{r}\left(T_{x_{0}} X \oplus V I_{f}\right)=T_{z} J_{I}^{r}(X, M)$ and $\operatorname{Ker} \Pi_{r}=\{0\} \oplus V I_{f}^{r}$. Moreover we have, for the Lagrange equivalence class

$$
[z]=\left\{j^{r} f^{\prime}\left(x_{0}^{\prime}\right) \mid x_{0}^{\prime} \in X, f^{\prime} \text { is Lagrange equivalent to } f\right\}
$$

in $J_{I}^{r}(X, M)$,

$$
T_{z}[z]=\Pi_{r}\left(T_{x_{0}} X \oplus\left(t f\left(m_{X} V_{X}\right)+w f\left(V L_{M}\right)\right)\right) .
$$

For the jet extension $j^{r} f:\left(X, x_{0}\right) \rightarrow J_{I}^{r}(n, M)$, we have

$$
\left(j^{r} f\right)_{*}\left(\frac{\partial}{\partial x_{i}}\right)=\Pi_{r}\left(\frac{\partial}{\partial x_{i}}, f_{*}\left(\frac{\partial}{\partial x_{i}}\right)\right) .
$$

Now the condition that $j^{r} f$ is transverse to $[z]=\left[j^{r} f\left(x_{0}\right)\right]$ at $x_{0}$ is equivalent to

$$
\left(j^{r} f\right)_{*}\left(T_{x_{0}} X\right)+T_{z}[z]=T_{z} J_{I}^{r}(X, M),
$$

and to the one that

$$
\left(\Pi_{r}\right)^{-1}\left(\left(j^{r} f\right)_{*}\left(T_{x_{0}} X\right)\right)+T_{x_{0}} X \oplus\left(t f\left(m_{X} V_{X}\right)+w f\left(V L_{M}\right)\right)+\{0\} \oplus V I_{f}^{r}
$$

coincides with $T_{x_{0}} X \oplus V I_{f}$. This condition is equivalent to

$$
V I_{f}=\left\langle f_{*}\left(\frac{\partial}{\partial x_{1}}\right), \ldots, f_{*}\left(\frac{\partial}{\partial x_{n}}\right)\right\rangle_{\mathbf{R}}+t f\left(m_{X} V_{X}\right)+w f\left(V L_{M}\right)+V I_{f}^{r},
$$

thus

$$
V I_{f}=t f\left(V_{X}\right)+w f\left(V L_{M}\right)+V I_{f}^{r}
$$

We recall that

$$
C_{I}^{\infty}(X, M)^{1}:=\left\{f \in C^{\infty}(X, M) \mid f \text { is isotropic and of corank } \leq 1\right\}
$$


is a Baire space ([13]). Furthermore we have

Theorem 4.1 ([13]). Let $Q$ be a submanifold of $J_{I}^{r}(X, M)$. Then the set

$$
T=\left\{f \in C_{I}^{\infty}(X, M)^{1} \mid j^{r} f: X \rightarrow J_{I}^{r}(X, M) \text { is transverse to } Q\right\}
$$

is residual and therefore dense in $C_{I}^{\infty}(X, M)^{1}$.

\section{Transversality and Lagrange stability}

Proof of Theorem 1.1. The equivalence of (hs), (is), (a) and $\left(\mathrm{a}^{\prime}\right)$ is already shown in [14]. We show the remaining implications.

(s) $\Rightarrow\left(\mathrm{t}_{r}\right)$ : Take a representative $f: U \rightarrow T^{*} Y$ of $f$ such that $f \in C_{I}^{\infty}(X, M)^{1}$. By Theorem 4.1, $f$ is approximated by $f^{\prime} \in C_{I}^{\infty}(X, M)^{1}$ such that $j^{r} f^{\prime}: U \rightarrow J_{I}^{r}(X, M)$ is transverse to the Lagrange orbit $\left[j^{r} f\left(x_{0}\right)\right]$. Since $f$ is Lagrange stable, there exists $x_{0}^{\prime} \in U$ such that $\left(f_{x_{0}^{\prime}}^{\prime}, \pi\right)$ and $\left(f_{x_{0}}, \pi\right)$ are Lagrange equivalent. Then $j^{r} f^{\prime}$ is transverse to $\left[j^{r} f\left(x_{0}\right)\right]$ at $x_{0}^{\prime}$, and therefore $j^{r} f$ is transverse to $\left[j^{r} f\left(x_{0}\right)\right]$ at $x_{0}$.

$\left(\mathrm{t}_{r}\right) \Rightarrow\left(\mathrm{a}_{r}^{\prime \prime}\right)$ : As we see in Section 4, condition $\left(\mathrm{t}_{r}\right)$ is equivalent to

$$
V I_{f}=t f\left(V_{X}\right)+w f\left(V L_{M}\right)+V I_{f}^{r} .
$$

Taking generating functions of both sides, we have

$$
R_{f}=(\pi \circ f)^{*} E_{Y}+\sum_{i=1}^{n}(\pi \circ f)^{*} E_{Y} p_{i} \circ f+R_{f} \cap m_{X}^{r+2} .
$$

Remarking $R_{f}=f^{*} E_{T^{*} Y}$, we have $\left(\mathrm{a}_{r}^{\prime \prime}\right)$.

$\left(\mathrm{a}_{r}^{\prime \prime}\right) \Rightarrow\left(\mathrm{a}^{\prime}\right)$ Since $R_{f} \cap m_{X}^{r+2} \subset m_{M}^{n+3} R_{f},\left(\mathrm{a}_{r}^{\prime \prime}\right)$ implies that $R_{f} /\left(m_{Y} R_{f}+m_{M}^{n+3} R_{f}\right)$ is generated by $1, p_{1} \circ f, \ldots, p_{n} \circ f$ over $\mathbf{R}$. Then we have $m_{M}^{n+1} R_{f} \subset m_{Y} R_{f}+m_{M}^{n+3} R_{f}$, therefore, by Nakayama's lemma, $m_{M}^{n+1} R_{f} \subset m_{Y} R_{f}$. Then $m_{Y} R_{f}+m_{M}^{n+3} R_{f}=m_{Y} R_{f}$, so we see that $R_{f} / m_{Y} R_{f}$ is generated by $1, p_{1} \circ f, \ldots, p_{n} \circ f$ over $\mathbf{R}$, namely, condition ( $\left.\mathrm{a}^{\prime}\right)$.

Thus we see the implication $\left(\mathrm{t}_{r}\right) \Rightarrow$ (is).

$\left(\mathrm{t}_{r}\right) \&$ (is) $\Rightarrow(\mathrm{s}):$ If $j^{r} f$ is transverse to $\left[j^{r} f\left(x_{0}\right)\right]$ at $x_{0}$, then there exists a neighbourhood $W \subset C_{I}^{\infty}(X, M)^{1}$ of an isotropic representative $f: U \rightarrow T^{*} Y$ such that, for any $f^{\prime} \in W, j^{r} f^{\prime}$ is transverse to $\left[j^{r} f\left(x_{0}\right)\right]$ at a point $x_{0}^{\prime} \in U$. Since $j^{r} f^{\prime}\left(x_{0}^{\prime}\right) \in\left[j^{r} f\left(x_{0}\right)\right]$, there exists an isotropic map-germ $f^{\prime \prime}:\left(X, x_{0}\right) \rightarrow T^{*} Y$ which is Lagrange equivalent to $f_{x_{0}^{\prime}}^{\prime}$ with respect to $\pi$ and $j^{r} f^{\prime \prime}\left(x_{0}\right)=j^{r} f\left(x_{0}\right)$. On the other hand, since $f$ is infinitesimally Lagrange stable, by Theorem 1.3, $f$ is strictly Lagrange $r$-determined. Therefore $\left(f^{\prime \prime}, \pi\right)$ and $(f, \pi)$ are Lagrange equivalent. Thus $\left(f_{x_{0}^{\prime}}^{\prime}, \pi\right)$ and $(f, \pi)$ are Lagrange equivalent, and $f$ is Lagrange stable.

\section{Supplementary remarks}

On the point (C): Let $f, f^{\prime}:\left(\mathbf{R}^{n}, 0\right) \rightarrow\left(T^{*} \mathbf{R}^{n}, 0\right)$ be Lagrange stable open Whitney umbrellas. Then we naturally ask whether the isomorphism $\left(Q_{f}, e\right) \cong\left(Q_{f^{\prime}}, e^{\prime}\right)$ implies that $f$ and $f^{\prime}$ are Lagrange equivalent or not. Here $Q_{f}=f^{*} E_{T^{*} \mathbf{R}^{n}} /(\pi \circ f)^{*} m_{\mathbf{R}^{n}} f^{*} E_{T^{*} \mathbf{R}^{n}}$ and $e$ is the generating function of $f$ with $e(0)=0$. In the case of complex analytic Lagrange immersions, the implication is true by Yau's theorem [25]. See also [19]. In real case, there exists a counterexample [8]. See also [21]. So the problem is how to change the formulation in the real case. 
Also in Legendre case, similar question can be posed, analogously to Mather-Yau type theorem. For Legendre immersions, it is solved affirmatively by Mather's theorem on $K$-versal unfoldings of functions and Arnol'd's theorem on Legendre singularities, even in real case. The author has been informed about this fact by S. Izumiya.

On the point (E): Lagrange immersions $X^{n} \rightarrow T^{*} Y, \operatorname{dim} X=\operatorname{dim} Y=n$, with Lagrange stable jets are dense in the space of Lagrange immersions, if $n \leq 5$ [4]. This is based on Arnol'd's classification of simple Lagrange singularities [1]. So we can say that the nice range for Lagrange immersions is $\{n \in \mathbf{N} \mid n \leq 5\}$.

The generic Lagrange classification is known, consisting of finite lists, of open Whitney umbrellas for $n \leq 3$, and in this case all germs are of $L$-corank $\leq 1$. See [12].

Then it is natural to ask whether $n=4,5$ belong to the nice range for Lagrange projections of open Whitney umbrellas. Ilia A. Bogaevski has posed to the author the necessity of the theory of simple singularities of open Whitney umbrellas.

Besides, in the case $n=4$, there appear generic isotropic map-germs of corank one and of $L$-corank two. See [13]. The generic classification is unknown for $n \geq 4$.

Then, as the first step of the classification, it is natural to ask about the existence of Lagrange stable projections of open Whitney umbrellas with $L$-corank $\geq 2$. This question was posed by V. M. Zakalyukin on the occasion of the author's talk of the workshop "Caustics" in Warszawa.

The following example, found on 23 June 1998, answers Zakalyukin's question. The example is based on Shcherbak's parametrisation of the variety of irregular orbits of the reflection group $H_{4}[22]$.

EXAmple 6.1. Let $x, y, z, w, \lambda$ be the coordinates of $\mathbf{R}^{5}$ and let

$$
f:\left(\mathbf{R}^{5}, 0\right) \rightarrow\left(T^{*} \mathbf{R}^{5}, 0\right)
$$

be a map-germ defined by

$$
\begin{gathered}
p_{1} \circ f=x, p_{2} \circ f=\frac{1}{2} y^{2}, p_{3} \circ f=-\frac{1}{2} x y^{2}, p_{4} \circ f=-\frac{1}{3} y^{3}, p_{5} \circ f=-x y^{3}, \\
q_{1} \circ f=\frac{1}{2} x^{2}+\frac{1}{2} y^{2} z+\lambda y^{3}, q_{2} \circ f=x z+y w+y^{2}+3 \lambda x y, \\
q_{3} \circ f=z, q_{4} \circ f=w, q_{5} \circ f=\lambda .
\end{gathered}
$$

Then $f$ is an open Whitney umbrella of type 1 , Lagrange stable with respect to the standard projection $\pi: T^{*} \mathbf{R}^{5} \rightarrow \mathbf{R}^{5}$ defined by $\pi(p, q)=q$, $\operatorname{corank}(f)=1$, and $L$ - $\operatorname{corank}(f)=2$. The generating function of $f$ is given by

$$
e=\frac{1}{3} x^{3}+\frac{1}{4} y^{4}+\frac{1}{2} x y^{2} z+\frac{1}{6} y^{3} w+\frac{3}{2} x y^{3} \lambda,
$$

and the set of singular points of $\pi \circ f$ by

$$
x w+2 x y+3 x^{2} \lambda-y z^{2}-6 y^{2} z \lambda-9 y^{3} \lambda^{2}=0,
$$

in the source $\left(\mathbf{R}^{5}, 0\right)$.

$$
\begin{aligned}
& \text { Proof. (1) Set } X=\left(\mathbf{R}^{5}, 0\right), Y=\left(\mathbf{R}^{5}, 0\right) \text {. Then } \\
& \qquad R_{f}=\left\{h \in E_{X} \mid d h \in E_{X} d\left(f^{*} E_{T^{*} Y}\right)\right\}=\left\{h \in E_{X} \mid \frac{\partial h}{\partial y} \in\langle y, w+3 \lambda x\rangle_{E_{X}}\right\},
\end{aligned}
$$


and $R_{f}$ is generated by

$$
x, z, w, \lambda, y^{2}, y^{3}, y(w+3 \lambda x), y^{2}(w+3 \lambda x)
$$

as differentiable algebra. Therefore we see $R_{f}=f^{*} E_{T^{*} Y}$. Moreover $\operatorname{codim} \Sigma\left(f_{\mathbf{C}}\right)=2$. Then, by [14], Proposition 5.1, page 230, and by considering the multiplicity of $f$, we conclude that $f$ is an open Whitney umbrella of type 1 .

(2) By a straightforward calculation, we see that

$$
Q_{f}=f^{*} E_{T^{*} Y} / m_{Y} f^{*} E_{T^{*} Y}=R_{f} /\left\langle x^{2}, y w+y^{2}+3 \lambda x y, z, w, \lambda\right\rangle_{R_{f}},
$$

and $Q_{f}$ is generated by $1, p_{1} \circ f, \ldots, p_{5} \circ f$. Therefore by Theorem $1.1, f$ is Lagrange stable.

Added on 3 December 1998: Recently, Ilia Bogaevski has given an example of Lagrange stable isotropic map-germ $f:\left(\mathbf{R}^{4}, 0\right) \rightarrow\left(T^{*} \mathbf{R}^{4}, 0\right)$ of corank one and of $L$-corank two. Moreover he has given the classification of simple stable Lagrange projections of the open Whitney umbrella of type 1 .

\section{References}

[1] V. I. Arnol'd, Normal forms of functions near degenerate critical points, the Weyl groups of $A_{k}, D_{k}, E_{k}$, and Lagrangian singularities (in Russian), Funktsional. Anal. i Prilozhen. 6 (1972), no. 4, 3-25; English transl.: Funct. Anal. Appl. 6 (1972), 254-272.

[2] V. I. Arnol'd, Lagrangian manifolds with singularities, asymptotic rays, and the open swallowtail (in Russian), Funktsional. Anal. i Prilozhen. 15 (1981), no. 4, 1-14; English transl.: Funct. Anal. Appl. 15 (1981), 235-246.

[3] V. I. Arnol'd, Singularities of Caustics and Wave Fronts, Math. Appl. (Soviet Ser.) 62, Kluwer Academic Publ., Dordrecht, 1990.

[4] V. I. Arnol'd, S. M. Guseŭn-Zade, A. N. Varchenko, Singularities of Differentiable Maps I, Monogr. Math. 82, Birkhäuser, Boston, 1985.

[5] J. Damon, The unfolding and determinacy theorems for subgroups of $\mathcal{A}$ and $\mathcal{K}, \mathrm{Mem}$. Amer. Math. Soc. 50 (1984), no. 306.

[6] A. B. Givental', Lagrangian imbeddings of surfaces and the open Whitney umbrella (in Russian), Funktsional. Anal. i Prilozhen. 20 (1986), no. 3, 35-41; English transl.: Funct. Anal. Appl. 20 (1986), 197-203.

[7] A. B. Givental', Singular Lagrangian manifolds and their Lagrangian mappings (in Russian), Itogi Nauki i Tekhniki, Akad. Nauk SSSR, Ser. Sovremennye Problemy Matematiki 33, VINITI, Moscow, 1988, 55-112; English transl.: J. Soviet Math. 52 (1990), 3246-3278.

[8] M. Golubitsky, V. Guillemin, Contact equivalence for Lagrangian manifolds, Adv. Math. 15 (1975), 357-387.

[9] G. Ishikawa, Families of functions dominated by distributions of $\mathcal{C}$-classes of map-germs, Ann. Inst. Fourier (Grenoble) 33 (1983), 199-217.

[10] G. Ishikawa, The local model of an isotropic map-germ arising from one dimensional symplectic reduction, Math. Proc. Cambridge Philos. Soc. 111 (1992), 103-112.

[11] G. Ishikawa, Parametrization of a singular Lagrangian variety, Trans. Amer. Math. Soc. 331 (1992), 787-798.

[12] G. Ishikawa, Parametrized Legendre and Lagrange varieties, Kodai Math. J. 17 (1994), 442-451. 
[13] G. Ishikawa, Transversalities for Lagrange singularities of isotropic mappings of corank one, in: Singularities and Differential Equations, S. Janeczko, W. Zajączkowski and B. Ziemian (eds.), Banach Center Publ. 33, Warsaw, 1996, 93-104.

[14] G. Ishikawa, Symplectic and Lagrange stabilities of open Whitney umbrellas, Invent. Math. 126 (1996), 215-234.

[15] S. Janeczko, Generating families for images of Lagrangian submanifolds and open swallowtails, Math. Proc. Cambridge Philos. Soc. 100 (1986), 91-107.

[16] B. Malgrange, Ideals of Differentiable Functions, Oxford Univ. Press, London, 1966.

[17] B. Malgrange, Frobenius avec singularités. 2. Le cas général, Invent. Math. 39 (1977), 67-89.

[18] J. N. Mather, Stability of $C^{\infty}$ mappings. I: The division theorem, Ann. of Math. (2) 87 (1968), 89-104. II: Infinitesimal stability implies stability, Ann. of Math. (2) 89 (1969), 254-291. III: Finitely determined map-germs, Inst. Hautes Études Sci. Publ. Math. 35 (1968), 127-156. IV: Classification of stable germs by $\mathbf{R}$ algebras, Inst. Hautes Études Sci. Publ. Math. 37 (1970), 223-248. V: Transversality, Adv. Math. 4 (1970), 301-336. VI: The nice dimensions, in: Proceedings of Liverpool Singularities-Symposium, I (1969/70), Lecture Notes in Math. 192, Springer, Berlin, 1971, 207-253.

[19] J. N. Mather, S. S.-T. Yau, Classification of isolated hypersurface singularities by their moduli algebras, Invent. Math. 69 (1982), 243-251.

[20] D. Mond, Deformations which preserve the non-immersive locus of a map-germ, Math. Scand. 66 (1990), 21-32.

[21] A. du Plessis, L. Wilson, On right-equivalence, Math. Z. 190 (1985), 163-205.

[22] O. P. Shcherbak, Wave fronts and reflection groups (in Russian), Uspekhi Mat. Nauk 43 (1988), no. 3, 125-160; English transl.: Russian Math. Surveys 43 (1988), no. 3, 149-194.

[23] T. Tsukada, unpublished note.

[24] C. T. C. Wall, Finite determinacy of smooth map-germs, Bull. London Math. Soc. 13 (1981), 481-539.

[25] S. S.-T. Yau, Criteria for right-left equivalence and right equivalence of holomorphic functions with isolated critical points, Proc. Sympos. Pure Math. 41 (1984), 291-297.

[26] V. M. Zakalyukin, Lagrangian and Legendre singularities (in Russian), Funktsional. Anal. i Prilozhen. 10 (1976), no. 1, 26-36; English transl.: Funct. Anal. Appl. 10 (1976), no. 1, $23-31$.

[27] V. M. Zakalyukin, Generating ideals of Lagrangian varieties, in: Theory of Singularities and its Applications, V. I. Arnol'd (ed.), Adv. Soviet Math. 1, Amer. Math. Soc., Providence, 1990, 201-210.

[28] V. M. Zakalyukin, R. M. Roberts, Stability of Lagrangian manifolds with singularities (in Russian), Funktsional. Anal. i Prilozhen. 26 (1992), no. 3, 28-34; English transl.: Funct. Anal. Appl. 26 (1992), 174-178. 